\title{
On the equation
}

$$
x(x+1) \ldots(x+k-1)=y(y+d) \ldots(y+(m k-1) d), m=1,2
$$

by

\section{N. Saradha (Bombay), T. N. Shorey (Bombay) and R. TiJdeman (Leiden)}

1. Introduction. For distinct positive integers $d_{1}$ and $d_{2}$ the authors [7] considered the equation

$$
\begin{aligned}
x\left(x+d_{1}\right) \ldots\left(x+(k-1) d_{1}\right)= & y\left(y+d_{2}\right) \ldots\left(y+(k-1) d_{2}\right) \\
& \text { in integers } x>0, y>0, k \geq 2 .
\end{aligned}
$$

There is no loss of generality in assuming that $d_{1}<d_{2}$ and $\operatorname{gcd}\left(x, y, d_{1}, d_{2}\right)$ $=1$. Then the authors proved that there exists an effectively computable number $C$ such that equation (1) implies that either

$$
\max (x, y, k) \leq C
$$

or

$$
x=k+1, \quad y=2, \quad d_{1}=1, \quad d_{2}=4 .
$$

In this paper we shall consider equation (1) with $d_{1}=1$ and $d_{2} \geq 2$, i.e.

$$
\begin{aligned}
x(x+1) \ldots(x+k-1)=y & (y+d) \ldots(y+(k-1) d) \\
& \text { in integers } x>0, y>0, k \geq 2
\end{aligned}
$$

where, for convenience, we write $d$ for $d_{2}$. We prove

Theorem 1. Assume equation (3). Then $y \leq k^{2} d^{2} / 12$. Moreover, if $k$ or $d$ is odd, then $y \leq k^{2} d^{2} / 24$.

If $d \leq k+1$, then there is a prime $p \leq d$ which divides $y$. By comparing the orders to which $p$ divides both sides of (3) we shall show

Theorem 2. Assume equation $(3)$ with $d \leq k+1$. Then $y \equiv 2(\bmod 4)$ and $d=2^{l}$ for some integer $l \geq 2$.

Equation (3) has no solutions for $d=2$, and infinitely many solutions for $d=4$ in view of (2). If $d$ is a higher power of 2 , then we can use the 
arguments of Section 4 of [7] to derive upper bounds for $k, x$ and $y$, but these bounds are unpractical.

We show for small values of $d$ how all solutions of (3) can be found in a practical way if $d$ has an odd prime divisor. It will be shown that for $k=2$ there exists a solution for any $d$ and that for $k=3$ there is a solution for any $d$ which is not divisible by 3 . We do not know any solution for $k \geq 4$ except for $(2)$ and $20 \times 21 \times 22 \times 23=7 \times 20 \times 33 \times 46$ due to M. Mirkowska and A. Mąkowski [4] and Ya. Gabovich [3], respectively. We shall compute all solutions for $d=2,3,5,6,7,9$ and 10 .

For positive integers $d_{1}$ and $d_{2}$, Saradha and Shorey [5], [6] considered the equation

$$
\begin{aligned}
x\left(x+d_{1}\right) \ldots\left(x+(k-1) d_{1}\right)= & y\left(y+d_{2}\right) \ldots\left(y+(2 k-1) d_{2}\right) \\
& \text { in integers } x>0, y>0, k \geq 2 .
\end{aligned}
$$

Saradha and Shorey [6] proved that equation (4) implies that either $\max (x, y, k) \leq C_{1}$ or $k=2, d_{1}=2 d_{2}^{2}, x=y^{2}+3 d_{2} y$ where $C_{1}$ is an effectively computable number depending only on $d_{1}$ and $d_{2}$. In this paper, we consider equation (4) with $d_{1}=1$. It was shown in [5] that equation (4) with $d_{1}=d_{2}=1$ has only one solution, namely, $(x, y, k)=(8,1,3)$. Therefore, we assume that $d_{1}=1$ and $d_{2} \geq 2$, i.e.

$$
\begin{aligned}
x(x+1) \ldots(x+k-1)=y(y+d) \ldots(y+(2 k-1) d) & \\
& \text { in integers } x>0, y>0, k \geq 2
\end{aligned}
$$

where, as earlier, we write $d$ for $d_{2}$. By pairing the factors on the right hand side of equation (5), we rewrite (5) as

$$
x(x+1) \ldots(x+k-1)=\prod_{j=0}^{k-1}\left(u+j(2 k-1-j) d^{2}\right),
$$

where

$$
u=y(y+(2 k-1) d) .
$$

Further, we define

$$
\theta= \begin{cases}3 & \text { if } k \equiv 1,2(\bmod 3) \text { or } d \equiv 0(\bmod 3), \\ 1 & \text { if } k \equiv 0(\bmod 6) \text { and } d \not \equiv 0(\bmod 3), \\ 2 & \text { if } k \equiv 3(\bmod 6) \text { and } d \neq \equiv 0(\bmod 3) .\end{cases}
$$

We shall prove the following results on equation (5) analogous to Theorems 1 and 2 .

Theorem 3. The equation (5) implies that

$$
u \leq(.44) k^{4} d^{4} / \theta .
$$

Theorem 4. The equation (5) with $d \leq k+1$ implies that $k \leq 35$ and that $d=2^{l}$ for some integer $l \geq 2$. 
We apply Theorems 3 and 4 to compute all solutions of equation (5) with $d=5,6$. For this and for computing all the solutions of equation (3) with $d=2,3,5,6,7,9,10$, a pocket calculator is sufficient. We assume tacitly that equation (3) holds in Sections 2-6 and that equation (5) holds in Sections 7-9.

2. Equation (3) with $d$ prime. The following simple observation turns out to be very useful.

Lemma 1. If $p$ is a prime with $p \mid d$ and $p \leq k+1$, then $p \mid y$.

Proof. If $p$ divides $d$ and the left hand side of (3), then it divides the right hand side and hence $y$.

If $p \leq k+1$ and $p$ does not divide the left hand side of (3), then $p=k+1$ and $x \equiv 1(\bmod p)$. It follows that the left hand side is $\equiv k !=(p-1) ! \equiv-1$ $(\bmod p)$. However, the right hand side of $(3)$ is $\equiv 0$ or $1(\bmod p)$. Since $p=k+1 \geq 3$ this is a contradiction.

LEMMA 2. If $d$ is prime, then $k \leq d-2$.

Proof. Suppose $d<k+2$. Then $d \mid y$ by Lemma 1 . Since $y<x$ and $y+(k-1) d>x+k-1$ in view of (3), all multiples of $d$ occurring on the left hand side of (3) also occur on the right hand side. Divide the common factors on both sides. Then the remaining left hand side is not divisible by $d$, whereas the remaining right hand side is a multiple of $d$.

Corollary $1 . d \geq 4$.

Proof. By Lemma 2 and $k \geq 2$.

3. Proof of Theorem 1. We use the same notation as in [7]. In particular $\underline{x}=x+(k-1) / 2, \bar{x}=x+k-1, y=y+(k-1) d / 2, \bar{y}=y+(k-1) d$.

LEMMA 3. $\underline{x} \leq \underline{y}-1 / 2$.

Proof. This is Lemma 1 of [7].

The following lemma is a refinement of Lemma 2 of [7] in case $d_{1}=1$, $d_{2}=d$.

Lemma 4. Put $h=\underline{y}-\underline{x}$. Let $h \geq \Delta>0$. If $k=2$, then

$$
\underline{y} \leq \frac{d^{2}-1}{8 \Delta}+\frac{\Delta}{2} \text {. }
$$

If $k=3$, then

$$
\underline{y} \leq \frac{d^{2}}{3 \Delta}+\Delta .
$$


If $k \geq 2$ and $d \geq \Delta \sqrt{6}$, then

$$
\underline{y}<\frac{k^{2} d^{2}}{24 \Delta}+k \Delta
$$

Proof. We shall use the fact that

$$
\underline{y}-\underline{x}=y-x+\frac{(k-1)(d-1)}{2}<\frac{(k-1)(d-1)}{2} .
$$

If $k=2$, then (3) implies $4 \underline{x}^{2}-1=4 \underline{y}^{2}-d^{2}$. Hence

$$
4 \underline{y}^{2}-d^{2} \leq 4(\underline{y}-\Delta)^{2}-1=4 \underline{y}^{2}-8 \underline{y} \Delta+4 \Delta^{2}-1,
$$

which implies (8). If $k=3$, then (3) implies $\underline{x}^{3}-\underline{x}=\underline{y}^{3}-d^{2} \underline{y}$. Hence

$$
\underline{y}^{3}-d^{2} \underline{y} \leq(\underline{y}-\Delta)^{3} \leq \underline{y}^{3}-3 \Delta \underline{y}^{2}+3 \Delta^{2} \underline{y},
$$

which implies (9).

For the proof of (10), we may assume that $k \geq 4$, otherwise (10) follows from (8) and (9). Suppose $\underline{y} \geq k^{2} d^{2} /(24 \Delta)+k \Delta$. Hence $\underline{y} \geq(1 / 6) \sqrt{6} k^{3 / 2} d$. As in the proof of Lemma 2 of [7] we have on the one hand, by $k^{2} \Delta \leq$ $k^{2} d^{2} /(6 \Delta)<\underline{y} / 4$,

$$
G_{2}(\underline{y})=G_{1}(\underline{x})<\underline{x}^{k} \leq(\underline{y}-\Delta)^{k} \leq \underline{y}^{k}-k \Delta \underline{y}^{k-1}+\frac{1}{2} k^{2} \Delta^{2} \underline{y}^{k-2}
$$

and, on the other hand,

$$
G_{2}(\underline{y}) \geq \underline{y}^{k}-\frac{1}{24} k\left(k^{2}-1\right) d^{2} \underline{y}^{k-2}-\sum_{\nu=3}^{[k / 2]} \frac{k^{3 \nu}}{2^{3 \nu} 3^{\nu}} d^{2 \nu} \underline{y}^{k-2 \nu},
$$

where

$$
G_{1}(Z)=\left(Z-\frac{k-1}{2}\right)\left(Z-\frac{k-3}{2}\right) \cdots\left(Z+\frac{k-1}{2}\right)
$$

and

$$
G_{2}(Z)=\left(Z-\frac{(k-1) d}{2}\right)\left(Z-\frac{(k-3) d}{2}\right) \ldots\left(Z+\frac{(k-1) d}{2}\right) .
$$

Combining both estimates we obtain

$$
\underline{y} \leq \frac{k \Delta}{2}+\frac{\left(k^{2}-1\right) d^{2}}{24 \Delta}+\frac{1}{k \Delta} \sum_{\nu=3}^{[k / 2]} \frac{k^{3 \nu}}{24^{\nu}} d^{2 \nu} \underline{y}^{2-2 \nu} .
$$

By our assumptions we have

$$
\frac{k^{3} d^{2}}{24 \underline{y}^{2}}=\frac{1}{4}\left(\frac{k^{3 / 2} d}{\sqrt{6} \underline{y}}\right)^{2} \leq \frac{1}{4} .
$$

Hence the last term of (11) is bounded by

$$
\frac{k^{8} d^{6}}{24^{3} \Delta \underline{y}^{4}} \sum_{\nu=0}^{\infty}\left(\frac{k^{3} d^{2}}{24 \underline{y}^{2}}\right)^{\nu} \leq\left(\frac{k^{2} d^{2}}{24 \Delta \underline{y}}\right)^{2}\left(\frac{k^{3 / 2} d}{\sqrt{6} \underline{y}}\right)^{2} \frac{k \Delta}{3} \leq \frac{k \Delta}{3} .
$$


So, by (11), we have $\underline{y}<k^{2} d^{2} /(24 \Delta)+k \Delta$.

COROLlary 2. We have

$$
\bar{x}=\underline{x}+\frac{k-1}{2} \leq \underline{y}+\frac{k}{2}-1<\frac{k^{2} d^{2}}{12}+k .
$$

Moreover, if $k$ or $d$ is odd, then

$$
\bar{x} \leq \underline{y}+\frac{k}{2}-1<\frac{k^{2} d^{2}}{24}+\frac{3 k}{2} .
$$

Proof. The first formula follows from Lemmas 3 and 4 by taking $\Delta=$ $1 / 2$. If $k$ or $d$ is odd, then $h \in \mathbb{Z}$. By Lemma 3 we can therefore take $\Delta=1$ in Lemma 4 . This yields the second upper bound for $\bar{x}$.

Proof of Theorem 1 . Put $\Delta=1 / 2$ and 1 , respectively, in (10) and use the fact that

by Corollary 1.

$$
y=\underline{y}-\frac{k-1}{2} d \leq \underline{y}-2(k-1)
$$

4. Proof of Theorem 2. In this section we elaborate the idea used in Section 2.

Lemma 5. Suppose (3) holds and $d$ is divisible by a prime $p$ which also divides $y$. Then

$$
k \operatorname{ord}_{p}(d)+\operatorname{ord}_{p}(k)<\frac{\log \bar{x}}{\log p} \quad \text { if } \operatorname{ord}_{p}(y) \geq \operatorname{ord}_{p}(d)
$$

and

$$
\begin{aligned}
k\left(\operatorname{ord}_{p}(y)-\frac{1}{p-1}\right)<k \operatorname{ord}_{p}(y)-\operatorname{ord}_{p}((k-1) !) & <\frac{\log \bar{x}}{\log p} \\
& \text { if } \operatorname{ord}_{p}(y)<\operatorname{ord}_{p}(d) .
\end{aligned}
$$

Proof. The power of $p$ dividing the left hand side of (3) is positive and at most

$$
\max _{0 \leq i<k} \operatorname{ord}_{p}(x+i)+\operatorname{ord}_{p}((k-1) !)<\frac{\log \bar{x}}{\log p}+\operatorname{ord}_{p}((k-1) !) .
$$

If $\operatorname{ord}_{p}(y) \geq \operatorname{ord}_{p}(d)$, then $p$ divides the right hand side at least to the power $k \operatorname{ord}_{p}(d)+\operatorname{ord}_{p}(k !)$. If $\operatorname{ord}_{p}(y)<\operatorname{ord}_{p}(d)$, then $p$ divides the right hand side exactly to the power $k \operatorname{ord}_{p}(y)$. Compare upper and lower bounds and use the fact that $\operatorname{ord}_{p}((k-1) !)<k /(p-1)$.

Proof of Theorem 2. We shall apply, without reference, several times Lemma 5 to obtain a lower bound for $\bar{x}$ and Corollary 2 to obtain an upper bound for $\bar{x}$ in the proof of Theorem 2. Assume that $d \leq k+1$. Let 
$p$ be a prime factor of $d$. Then $p \mid y$ by Lemma 1 . If $p \geq 5$, then

$$
5^{3 k / 4} \leq p^{k(1-1 /(p-1))}<\bar{x}<\frac{k^{2} d^{2}}{12}+k \leq \frac{k^{2}(k+1)^{2}}{12}+k,
$$

which yields a contradiction for all $k \geq 2$.

If $p=3$, then

$$
3^{k}<\bar{x}<\frac{k^{2}(k+1)^{2}}{12}+k \quad \text { if } \operatorname{ord}_{3}(y) \geq \operatorname{ord}_{3}(d),
$$

which gives a contradiction for all $k \geq 2$, and

$$
3^{k\left(\operatorname{ord}_{3}(y)-1 / 2\right)}<\bar{x}<\frac{k^{2}(k+1)^{2}}{12}+k \quad \text { if } \operatorname{ord}_{3}(y)<\operatorname{ord}_{3}(d) .
$$

In the latter case $\operatorname{ord}_{3}(y)=1$ and $9 \mid d$ and $k \leq 16$. Since $d \leq k+1$, we have $d=9$. Hence,

$$
3^{k-\operatorname{ord}_{3}((k-1) !)}<\bar{x}<\frac{27 k^{2}}{8}+\frac{3 k}{2},
$$

which is impossible, since $k \geq d-1=8$.

If $p=2$ and $4 \nmid d$, then

$$
2^{k+\operatorname{ord}_{2}(k)}<\bar{x}<\frac{k^{2} d^{2}}{12}+k \leq \frac{k^{2}(k+1)^{2}}{12}+k .
$$

This implies $k \leq 9$. Hence, we derive from Corollary 1 that $d \in\{6,10\}$, but these cases were treated when dealing with $p=3$ and $p=5$.

If $p=2$ and $4 \mid d$, then either $\operatorname{ord}_{2}(y)=1$ or

$$
2^{k+1} \leq 2^{2 k-\operatorname{ord}_{2}((k-1) !)}<\bar{x}<\frac{k^{2} d^{2}}{12}+k \leq \frac{k^{2}(k+1)^{2}}{12}+k .
$$

The latter case is impossible for all $k \geq 2$. Thus $d$ has no odd prime divisor, $4 \mid d$ and $y \equiv 2(\bmod 4)$.

5. Equation (3) with $k=2$ and $k=3$. Let $d$ be fixed. If $k=2,3$ all solutions of (3) in integers $x>0$ and $y>0$ can be found by the following algorithms.

Algorithm for $k=2$. For $h=[\sqrt{d}-3 / 2]+1, \ldots,[(d-2) / 2]$ compute $y=$ $\left(h^{2}+h\right) /(d-2 h-1)$. If $y \in \mathbb{Z}$, then a solution is given by $(x, y)=(y+h, y)$.

Proof. Suppose $x(x+1)=y(y+d)$. Put $x=y+h$. Then $h>0$ and $2 y h+h^{2}+y+h=y d$. Hence $y=\left(h^{2}+h\right) /(d-2 h-1)$. Note that $d-2 h-1 \leq h^{2}+h$ whence $h>\sqrt{d}-3 / 2$ and that $2 h<d-1$ whence $h \leq(d-2) / 2$.

R e mark. If $d$ is even, then $h=(d-2) / 2$ yields the solution

$$
(x, y)=\left(\frac{d^{2}-4}{4}, \frac{d^{2}-2 d}{4}\right) \text {. }
$$


If $d$ is odd, then $h=(d-3) / 2$ yields the solution

$$
(x, y)=\left(\frac{d^{2}-9}{8}, \frac{d^{2}-4 d+3}{8}\right) .
$$

Algorithm for $k=3$. For $h=1,2, \ldots, d-2$ check whether $\left(d^{2}+3 h^{2}-1\right)^{2}-$ $12 h^{2}\left(h^{2}-1\right)$ is a square, $D^{2}$ say. If so, check whether $\left(d^{2}+3 h^{2}-1 \pm D\right) /(6 h)$ is an integer $\underline{y}$ with $\underline{y}>d$. If so, then $(x, y)=(\underline{y}-h-1, \underline{y}-d)$ provides a solution.

Pr o o f. Suppose $x(x+1)(x+2)=y(y+d)(y+2 d)$. Then $\underline{x}^{3}-\underline{x}=\underline{y}^{3}-d^{2} \underline{y}$. Put $\underline{y}=\underline{x}+h$. Then $h>0$ and $3 h \underline{y}^{2}-\left(d^{2}+3 h^{2}-1\right) \underline{y}+h^{3}-h=\overline{0}$. Hence

$$
\underline{y}=\frac{d^{2}+3 h^{2}-1 \pm D}{6 h} \quad \text { where } D=\sqrt{\left(d^{2}+3 h^{2}-1\right)^{2}-12 h^{2}\left(h^{2}-1\right)} .
$$

Re mark. If $d$ is not a multiple of 3 , then $h=1$ leads to the solution

$$
(x, y)=\left(\frac{d^{2}-4}{3}, \frac{d^{2}-3 d+2}{3}\right) .
$$

6. Complete solutions of equation (3) for small values of $d$. We assume that (3) holds. We deal with $d$ satisfying $d \leq 10$. We determine all the solutions for the $d$ 's which are not perfect powers of 2 . For the treatment of powers of 2 we refer to Section 4 of [7]. Extensive computation would be needed to determine all solutions in that way.

Case $d=4$. By Lemma 1, $y$ is even. By Lemma 5 and Corollary 2,

$$
2^{2 k}<\bar{x}<\frac{4 k^{2}}{3}+k \quad \text { if } \operatorname{ord}_{2}(y) \geq 2,
$$

which is impossible for $k \geq 2$. Thus $y \equiv 2(\bmod 4)$. For every $k$ there is a solution $(k, x, y)=(k, k+1,2)$ according to (2). The (finitely many) other solutions can be determined by the method described in [7], but none is known.

C a s e $d=5$. By Theorem 2 we have $k \leq 3$. According to the algorithms in the previous section all solutions are given by $(k, x, y)=(2,2,1),(3,7,4)$.

Case $d=6$. By Theorem 2 we have $k \leq 4$. Hence, by Lemma $1,3 \mid y$. Then, by Lemma 5 and Corollary 2 ,

$$
3^{k+\operatorname{ord}_{3}(k)}<\bar{x}<3 k^{2}+k .
$$

This is impossible for $k=3,4$. For $k=2$ the algorithm yields one solution, namely $(x, y)=(8,6)$.

Case $d=7$. By Theorem 2 , we have $k \leq 5$. Suppose $7 \mid y$. Then, by (12) 
and Corollary 2, we have

$$
7^{k}<\bar{x}<\frac{49}{24} k^{2}+\frac{3}{2} k
$$

which has no solutions. Hence $7 \nmid y$. For $k=2$, the algorithm yields $(x, y)=$ $(5,3)$ and for $k=3$ we find $(x, y)=(4,1)$ and $(15,10)$. If $k=4$, then $(10)$ gives $y<37$ if $h=1$ and $y<25$ if $h \geq 2$. We further know that $x \equiv 1,2,3$ $(\bmod 7)$ and $h \leq 8$. Now, it is easy to exclude all the possibilities by looking at the prime factors of the left hand side and the right hand side of equation (3). If $k=5$, then (10) gives $\underline{y} \leq 56$ if $h=1$ and $\underline{y} \leq 35$ if $h \geq 2$. We further know that $x \equiv 1$ or $2(\bmod 7)$. All the possibilities are excluded again as mentioned in the case $k=4$.

C a s e $d=8$. By Lemma 1, $y$ is even. Hence, by Lemma 5 and Corollary 2 ,

$$
2^{3 k}<\bar{x}<\frac{16}{3} k^{2}+k \quad \text { if } \operatorname{ord}_{2}(y) \geq 3,
$$

which is impossible, and

$$
2^{k \operatorname{ord}_{2}(y)-\operatorname{ord}_{2}((k-1) !)}<\underline{x}<\frac{16}{3} k^{2}+k \quad \text { if } \operatorname{ord}_{2}(y)<3 .
$$

The algorithms for $k=2$ and 3 yield the solutions $(k, x, y)=(2,4,2)$, $(2,15,12)$ and $(3,20,14)$. So suppose $k \geq 4$. If $\operatorname{ord}_{2}(y)=2$, then

$$
2^{2 k-\operatorname{ord}_{2}((k-1) !)}<\frac{16}{3} k^{2}+k,
$$

which implies $k=5$. Then, by Corollary 2 ,

$$
2^{7}<\frac{8}{3} k^{2}+\frac{3}{2} k<75
$$

which is false. It remains to consider the case $\operatorname{ord}_{2}(y)=1, k \geq 4$. At least in principle, this can be done by following [7].

Case $d=9$. By Lemma 1 , we have $3 \mid y$. Hence, by Lemma 5 and Corollary 2, either

or

$$
3^{2 k}<\bar{x}<\frac{27}{8} k^{2}+\frac{3}{2} k \quad \text { and } \quad \operatorname{ord}_{3}(y) \geq 2
$$

$$
3^{k-\operatorname{ord}_{3}((k-1) !)}<\bar{x}<\frac{27}{8} k^{2}+\frac{3}{2} k \quad \text { and } \quad \operatorname{ord}_{3}(y)=1 .
$$

The former case is impossible, the latter is possible only if $k=2,10 \leq \bar{x} \leq 16$ or $k=3,28 \leq \bar{x} \leq 34$ or $k=4,28 \leq \bar{x} \leq 59$ or $k=5,82 \leq \bar{x} \leq 91$.

Note that $\operatorname{ord}_{3}(x(x+1)(x+2) \ldots(x+k-1))=k$. Using this it is easy to show that the only solution is given by $(k, x, y)=(2,9,6)$.

Case $d=10$. By Lemma $1, y$ is even and $k \leq 3$ or $5 \mid y$. The algorithms for $k=2$ and 3 yield the solutions $(k, x, y)=(2,7,4),(2,24,20)$ and 
$(3,32,24)$. Now suppose $k \geq 4$. Then $10 \mid y$. By Lemma 5 and Corollary 2 ,

$$
5^{k+\operatorname{ord}_{5}(k)}<\bar{x}<\frac{25}{3} k^{2}+k .
$$

This has no solution. So there is no solution other than the three stated above.

7. Proof of Theorem 3. We define rational integers $A_{1}^{\prime}, \ldots, A_{k-1}^{\prime}$ and $B_{1}^{\prime}, \ldots, B_{k-1}^{\prime}$ as

$$
x(x+1) \ldots(x+k-1)=x^{k}+A_{1}^{\prime} x^{k-1}+\ldots+A_{k-1}^{\prime} x
$$

and

$$
\prod_{j=0}^{k-1}\left(u+j(2 k-1-j) d^{2}\right)=u^{k}+B_{1}^{\prime} d^{2} u^{k-1}+\ldots+B_{k-1}^{\prime} d^{2 k-2} u .
$$

It follows from the definitions of $A_{i}^{\prime}$ and $B_{j}^{\prime}$ that

$$
A_{i}^{\prime} \leq k^{2 i} /\left(2^{i} i !\right), \quad B_{j}^{\prime} \leq(2 k)^{j} A_{j}^{\prime} \quad \text { for } 1 \leq i, j \leq k-1,
$$

implying

$$
B_{j}^{\prime} \leq k^{3 j} / j \text { ! for } 1 \leq j \leq k-1 .
$$

We put $F(z)=z(z+1) \ldots(z+k-1)$ and

$$
f=\frac{(k-1)(2 k-1) d^{2}}{3}-\frac{k-1}{2},
$$

and write $F(z+f)=z^{k}+A_{1}^{\prime}(f) z^{k-1}+\ldots+A_{k-1}^{\prime}(f) z+A_{k}^{\prime}(f)$, where

$$
A_{i}^{\prime}(f)=\left(\begin{array}{c}
k \\
i
\end{array}\right) f^{i}+\left(\begin{array}{c}
k-1 \\
i-1
\end{array}\right) f^{i-1} A_{1}^{\prime}+\ldots+\left(\begin{array}{c}
k-i+1 \\
1
\end{array}\right) f A_{i-1}^{\prime}+A_{i}^{\prime}
$$
for $1 \leq i \leq k$.

We note that $A_{i}^{\prime}$ and $B_{i}^{\prime}$ are obtained from $A_{i}$ and $B_{i}$ respectively by replacing $k-1$ for $k$ in [3, Lemma 11]. Thus, we obtain

LEMMA 6.

$$
\begin{gathered}
A_{1}^{\prime}=k(k-1) / 2, \quad A_{2}^{\prime}=k(k-1)(k-2)(3 k-1) / 24, \\
A_{3}^{\prime}=k^{2}(k-1)^{2}(k-2)(k-3) / 48
\end{gathered}
$$

and

$$
\begin{aligned}
B_{1}^{\prime}= & k(k-1)(2 k-1) / 3, \\
B_{2}^{\prime}= & k(k-1)\left(20 k^{4}-64 k^{3}+61 k^{2}-29 k+6\right) / 90, \\
B_{3}^{\prime}= & k(k-1)\left(280 k^{7}-1988 k^{6}+5218 k^{5}-6815 k^{4}\right. \\
& \left.+5176 k^{3}-2321 k^{2}+738 k-144\right) / 5670 .
\end{aligned}
$$

By Lemma 6, (14) and (15), we calculate the following relations. 
Lemma 7. (a) We have

$$
\left(\begin{array}{l}
k \\
2
\end{array}\right)\left(\frac{(k-1)(2 k-1) d^{2}}{3}\right)^{2}-B_{2}^{\prime} d^{4}<\frac{2}{45} k^{5} d^{4}
$$

and

$$
\left(\begin{array}{l}
k \\
3
\end{array}\right)\left(\frac{(k-1)(2 k-1) d^{2}}{3}\right)^{3}-B_{3}^{\prime} d^{6}<\frac{4}{135} k^{8} d^{6} .
$$

(b) For $k \geq 3$,

$A_{1}^{\prime}(f)-B_{1}^{\prime} d^{2}=0, \quad A_{2}^{\prime}(f)-B_{2}^{\prime} d^{4}>\frac{1}{30} k^{5} d^{4}, \quad A_{3}^{\prime}(f)-B_{3}^{\prime} d^{6}>0$.

In the following lemma, we give necessary and sufficient conditions for solving equation (5) with $k=2$.

LEMMA 8. Let $k=2$. For every integer $h$ with $1 \leq h \leq d^{2}-1$, suppose the following conditions (i) and (ii) hold.

(i) $h(h+1) /\left(2 d^{2}-2 h-1\right)=u$ is an integer,

(ii) $9 d^{2}+4 u$ is a square, say $D^{2}$.

Then

$$
(x, y)=(u+h,(D-3 d) / 2)
$$

is a solution of equation (5). Further, any solution $(x, y)$ in positive integers of equation (5) satisfies (i) and (ii) for some $h$ with $1 \leq h \leq d^{2}-1$ and (16).

Pro of. Suppose that (i) and (ii) are satisfied.Then $D-3 d$ is even. Let $x=u+h$ and $y=(D-3 d) / 2$. Then $y(y+3 d)=u$ and $(u+h)(u+h+1)=$ $u\left(u+2 d^{2}\right)$, which implies that $(x, y)$ is a solution of equation (5). Let $x>0$ and $y>0$ be integers satisfying equation (5). Then, by putting $y(y+3 d)=u$, we get $x(x+1)=u\left(u+2 d^{2}\right)$, which implies that $u<x<u+d^{2}$. Now set $x=u+h$. Then $1 \leq h \leq d^{2}-1$ and $u=h(h+1) /\left(2 d^{2}-2 h-1\right)$ is an integer. Now, the assertion follows from $y(y+3 d)=u$.

Proof of Theorem 3 . We assume (6) with

$$
u \geq(.44) k^{4} d^{4} / \theta
$$

and we shall arrive at a contradiction. We apply arithmetic-geometric mean to both sides of (6) to obtain

$$
x(x+1) \ldots(x+k-1)<\left(x+\frac{k-1}{2}\right)^{k}
$$

and

$$
\prod_{j=0}^{k-1}\left(u+j(2 k-1-j) d^{2}\right)<\left(u+\frac{(k-1)(2 k-1) d^{2}}{3}\right)^{k}
$$


Further, we observe from (6) and (19) that

$$
u<x<u+\frac{(k-1)(2 k-1) d^{2}}{3} .
$$

By (20), (17) and (7), we have

$$
x>u>(.146) k^{4} d^{4} .
$$

By Lemma 8, we derive that equation (5) with $k=2, d=2$ is not possible.

Thus, we assume that $k \geq 3$ whenever $d=2$. Suppose now

$$
x(x+1) \ldots(x+k-1) \leq\left(x+\frac{k-1}{2}-\frac{1}{6}\right)^{k} .
$$

Then

$$
\frac{k}{6} x^{k-1} \leq\left(\left(\begin{array}{l}
k \\
2
\end{array}\right)\left(\frac{k-1}{2}\right)^{2}-A_{2}^{\prime}\right) x^{k-2}+\left(\begin{array}{l}
k \\
3
\end{array}\right)\left(\frac{k-1}{2}\right)^{3} x^{k-3}+\ldots,
$$

which, by Lemma 6 and (20), implies that $x \leq k^{2} / 4+k^{5} /(4 x)$ and we obtain a contradiction from (21). Hence

$$
x(x+1) \ldots(x+k-1)>\left(x+\frac{k-1}{2}-\frac{1}{6}\right)^{k} .
$$

We now show that

$$
\prod_{j=0}^{k-1}\left(u+j(2 k-1-j) d^{2}\right)>\left(u+\frac{(k-1)(2 k-1) d^{2}-\theta / 2}{3}\right)^{k} .
$$

Suppose (23) does not hold. Then we see from Lemma 6 that

$$
\begin{aligned}
\frac{\theta k}{6} u^{k-1} \leq & \left(\left(\begin{array}{l}
k \\
2
\end{array}\right)\left(\frac{(k-1)(2 k-1) d^{2}}{3}\right)^{2}-B_{2}^{\prime} d^{4}\right) u^{k-2} \\
& +\left(\left(\begin{array}{l}
k \\
3
\end{array}\right)\left(\frac{(k-1)(2 k-1) d^{2}}{3}\right)^{3}-B_{3}^{\prime} d^{6}\right) u^{k-3} \\
& +\left(\begin{array}{l}
k \\
4
\end{array}\right)\left(\frac{(k-1)(2 k-1) d^{2}}{3}\right)^{4}\left(1+\frac{2 k^{3} d^{2}}{15 u}+\ldots\right) u^{k-4} .
\end{aligned}
$$

Since $2 k^{3} d^{2} /(15 u)<1 / 13$ by $(21)$ and $k \geq 3$ if $d=2$, we apply Lemma 7 (a) to the above inequality to obtain

$$
\frac{\theta k}{6} u^{k-1} \leq \frac{2}{45} k^{5} d^{4} u^{k-2}+\frac{4}{135} k^{8} d^{6} u^{k-3}+\frac{13}{1458} k^{12} d^{8} u^{k-4},
$$

which implies that

$$
u \leq \frac{k^{4} d^{4}}{\theta}\left(\frac{4}{15}+\frac{8}{45} \cdot \frac{k^{3} d^{2}}{u}+\frac{13}{243} \cdot \frac{k^{7} d^{4}}{u^{2}}\right) .
$$


By (21) and $k \geq 3$ if $d=2$, we derive from the above inequality that $u \leq(.422) k^{4} d^{4} / \theta$, which contradicts (17). This proves (23).

From (6), (18), (19), (22), (23) and (17), we conclude that $-\theta / 6<x-$ $u-f<1 / 6$, which by (7) and Liouville's inequality implies that

$$
x=u+f .
$$

Then $f$ is an integer and we derive from $(14)$ that either $k \equiv 1,5(\bmod 6)$ or $k \equiv 3(\bmod 6), d \equiv 0(\bmod 3)$, which implies that $k \geq 5$ if $d=2$ and $k \geq 3$ if $d \geq 3$, which we utilise in the subsequent argument of the proof of Theorem 3. We put (24) in (6) to obtain

$$
\begin{aligned}
A_{1}^{\prime}(f) u^{k-1}+A_{2}^{\prime}(f) u^{k-2}+ & A_{3}^{\prime}(f) u^{k-3} \\
& \leq B_{1}^{\prime} d^{2} u^{k-1}+B_{2}^{\prime} d^{4} u^{k-2}+B_{3}^{\prime} d^{6} u^{k-3}+\ldots
\end{aligned}
$$

Now, we use Lemma 7(b) to obtain

$$
\frac{1}{30} k^{5} d^{4} u^{k-2} \leq \frac{1}{24} k^{12} d^{8} u^{k-4}\left(1+\frac{k^{3} d^{2}}{5 u}+\ldots\right)
$$

and since $k^{3} d^{2} /(5 u)<1 / 14$ by $(21)$, we obtain $u^{2} \leq(1.35) k^{7} d^{4}$. This contradicts $(21)$.

8. Proof of Theorem 4. We begin with a lemma that gives a lower bound for $u$.

Lemma 9. Suppose $d \leq k+1$. If $p$ is a prime dividing $d$, then

$$
u>p^{2 k \operatorname{ord}_{p}(d)+\operatorname{ord}_{p}(k(k+1) \ldots(2 k))}-k^{4} \quad \text { if } \operatorname{ord}_{p}(y) \geq \operatorname{ord}_{p}(d)
$$

and

$$
u>p^{2 k \operatorname{ord}_{p}(y)-\operatorname{ord}_{p}((k-1) !)}-k^{4} \quad \text { if } \operatorname{ord}_{p}(y)<\operatorname{ord}_{p}(d) .
$$

Proof. First we follow the proof of Lemma 1 to derive that $p \mid y$. Then, as in Lemma 5 , we count the power of $p$ on both sides of equation (5) to obtain

$$
x+k-1 \geq p^{2 k \operatorname{ord}_{p}(d)+\operatorname{ord}_{p}(k(k+1) \ldots(2 k))} \quad \text { if } \operatorname{ord}_{p}(y) \geq \operatorname{ord}_{p}(d)
$$

and

$$
x+k-1 \geq p^{2 k \operatorname{ord}_{p}(y)-\operatorname{ord}_{p}((k-1) !)} \quad \text { if } \operatorname{ord}_{p}(y)<\operatorname{ord}_{p}(d) .
$$

We combine the above two inequalities with (20) and use the inequality $k+(k-1)(2 k-1) d^{2} / 3<k^{4}$ to obtain (25) and (26).

Proof of Theorem 4. We assume that

$$
d \leq k+1 \text {. }
$$

First, we consider the case where $d \neq 2^{l}$ with $l \geq 2$. Suppose $d$ is divisible by a prime $p \geq 5$. Then, by (27), we see that $k \geq 4$. We derive from Lemma 9 
that

$$
u>5^{(7 k+1) / 4}-k^{4} .
$$

By Theorem 3, (7) and (27), it follows that

$$
u<(.44) k^{4} d^{4}<(.44) k^{4}(k+1)^{4},
$$

which, since $k \geq 4$, contradicts (28). Thus, we may suppose that 3 is the largest prime dividing $d$. Then from Lemma 9 we obtain

$$
u>3^{(3 k+1) / 2}-k^{4},
$$

which contradicts (29) for $k \geq 12$. Let $\operatorname{ord}_{3}(d) \geq 2$. Then, by (27) and (7), we may assume that $d=9, k \in\{8,9,10,11\}$ and $\theta=3$. In these cases, we have $u>3^{2 k-\operatorname{ord}_{3}((k-1) !)}-k^{4}$, which contradicts Theorem 3 . Thus, we conclude that either $\operatorname{ord}_{3}(d)=1$ or $d=2$. Let $\operatorname{ord}_{3}(d)=1$. From (25), we obtain $u>3^{2 k+\operatorname{ord}_{3}(k \cdot \ldots \cdot 2 k)}-k^{4}$. This contradicts (29) for $k \geq 5$. Thus, by (27) and (7), we may suppose that $d=3, k \in\{2,3,4\}$ and $\theta=3$. Now, we apply Theorem 3 in these cases to contradict the preceding lower bound for $u$. Let $d=2$. We use (25) to obtain $u>2^{3 k}-k^{4}$, which contradicts Theorem 3 with $k \geq 4, \theta \geq 1, k=3, \theta=2$ and $k=2, \theta=3$.

Next, we turn to the case $d=2^{l}$ with $l \geq 2$. Then $l \leq[\log (k+1) / \log 2]$ by (27) and we apply Lemma 9 and Theorem 3 to derive that

$$
\frac{(.44)}{\theta} k^{4} 2^{4[\log (k+1) / \log 2]}>u>2^{2 k-\operatorname{ord}_{2}((k-1) !)}-k^{4},
$$

which implies that $k \leq 35$.

\section{Complete solutions of equation (5) for $d=5,6$}

Case $d=5$. By Theorem $4, k \leq 3$. If $k=2$, we apply Lemma 8 to derive that $(32,1)$ and $(207,8)$ are the only solutions. Let $k=3$. Then $x(x+1)(x+2)=u(u+100)(u+150)$ with $u=y(y+25)$ and $u<x \leq u+83$ by (20). We put $x=u+h$. Then $1 \leq h \leq 83$ and

$$
u^{2}(250-3 h-3)+u\left(15000-3 h^{2}-6 h-2\right)=h(h+1)(h+2) .
$$

For $h=83$, the right hand side of (30) is positive while the left hand side is negative. For $1 \leq h \leq 69$, we observe that the coefficient of $u$ in (30) is positive to derive that $40 u^{2}<69 \cdot 70 \cdot 71$, which implies that $u \leq 92$ and hence $y \leq 3$. We check that equation (5) with $y \in\{1,2,3\}$ is not satisfied. For $70 \leq h \leq 82$, we check that

$$
\left(15000-3 h^{2}-6 h-2\right)^{2}+4 h(h+1)(h+2)(250-3 h-3)
$$

is not a perfect square. Hence equation (5) admits only two solutions, namely, $(k, x, y)=(2,32,1)$ and $(2,207,8)$. 
Case $d=6$. By Theorem $4, k \leq 4$. If $k=2$, we check from Lemma 8 that there is no solution. Let $k=3$. Since $d$ is divisible by 3 and the left hand side of equation (5) is divisible by 3 , we observe that $3 \mid y$. We count the power of 3 on both sides of equation (5) as in the proof of (25) to derive that $x \geq 3^{8}-2$ and further, we have $u<x \leq u+119$. We put $x=u+h$ in (6) for $1 \leq h \leq 119$ to obtain

$$
(360-3 h-3) u^{2}+\left(31104-3 h^{2}-6 h-2\right) u=h(h+1)(h+2) .
$$

For $h=119$, the right hand side of (31) is positive while the left hand side is negative. For $1 \leq h \leq 118$, the left hand side of (31) exceeds $3 u^{2}-11378 u$ while the right hand side is at most $118 \cdot 119 \cdot 120$ and this implies that $u \leq 6000$, which is a contradiction. Hence, equation (5) with $k=3$ has no solution. Let $k=4$. Once again, we have $3 \mid y$ and $x \geq 3^{9}-3$. By Theorem 3 , $u \leq 48660$ whence, by $(20), x \leq 48911$. Since $x(x+1)(x+2)(x+3)$ is divisible by $3^{10}$ and $3^{10}>48911$, we see that $x$ or $x+3$ equals $3^{9}$ or $2 \cdot 3^{9}$. It is easy to check that these four cases cannot occur. Thus there is no solution when $d=6$.

10. Solutions of equation (5) for $k=2$. Fermat stated that there are no four squares in arithmetic progression. Euler's proof of this fact reveals that the product of four positive integers in arithmetic progression cannot be a square (cf. Dickson [2], p. 635). An obvious generalisation would be that the product of the terms of a finite arithmetic progression of length $\geq 4$ cannot be a square or another perfect power. Such questions have occupied various authors. See e.g. Shorey and Tijdeman [8]. An extension in another direction would be to show that the product of four positive integers in arithmetic progression cannot be the product of two consecutive integers. This is the special case $k=2$ of equation (4),

$$
x(x+1)=y(y+d)(y+2 d)(y+3 d) .
$$

For given $d$ all solutions $x, y$ can be found by Lemma 8. In Section 9 it has been shown that there are two solutions in case $d=5$ and none if $d=6$. A computer search yields the following solutions for $d<100$ :

\begin{tabular}{llllll}
\hline$d$ & $h$ & $u$ & $D$ & $x$ & $y$ \\
\hline 5 & 16 & 16 & 17 & 32 & 1 \\
5 & 23 & 184 & 31 & 207 & 8 \\
23 & 527 & 92752 & 613 & 93279 & 272 \\
74 & 5264 & 65520 & 558 & 70784 & 168 \\
77 & 3952 & 3952 & 263 & 7904 & 16 \\
88 & 3644 & 1620 & 276 & 5264 & 6 \\
\hline
\end{tabular}


Note that $h=u$ occurs for $d=5$ and $d=77$. The assumption $h=u$ in Lemma 8 yields that $3 h=2 d^{2}-2$ and that $\frac{35}{3} d^{2}-\frac{8}{3}$ is a square of an integer. This yields the Pell equation $35 d^{2}-3 D^{2}=8$. The already found solutions $(d, D)=(5,17)$ and $(77,263)$ lead to the parametrised solutions

$$
\begin{aligned}
(-9+\sqrt{105})(41+4 \sqrt{105})^{n} & =3 D_{n}+d_{n} \sqrt{105} & & (n=1,2, \ldots) \\
(9+\sqrt{105})(41+4 \sqrt{105})^{n} & =3 D_{n}^{\prime}+d_{n}^{\prime} \sqrt{105} & & (n=1,2, \ldots) .
\end{aligned}
$$

This shows that (32) has infinitely many solutions $(d, x, y)$. For $n=1$ we find the known solutions $\left(d_{1}, D_{1}\right)=(5,17)$ and $\left(d_{1}^{\prime}, D_{1}^{\prime}\right)=(77,263)$. For $n=2$ we obtain $\left(d_{2}, D_{2}\right)=(409,1397)$ and $\left(d_{2}^{\prime}, D_{2}^{\prime}\right)=(6313,21563)$ leading to the new solutions $(d, x, y)=(409,223040,85),(6313,53138624,1312)$ of (32).

The assumption $u=c h$ for some fixed number $c$ will lead to infinite classes of solutions in the same way as $u=h$ does. E.g. for $c=8$ we find the Pell equation $665 d^{2}-17 D^{2}=288$. We know from the list that $(d, D)=(5,31)$ is a solution and hence there are infinitely many.

It is obvious that (32) admits only finitely many solutions $(y, d)$ for fixed $x$ and the algorithm shows that there are only finitely many solutions $(x, y)$ for fixed $d$. For fixed $y$, equation (32) has only finitely many solutions $(d, x)$, since (32) is equivalent to the elliptic equation

$$
Y^{2}=24 y X^{3}+44 y^{2} X^{2}+24 y^{3} X+4 y^{4}+1,
$$

where $X=d$ and $Y=2 x+1$. See Baker [1].

\section{References}

[1] A. Baker, Bounds for the solutions of the hyperelliptic equation, Proc. Cambridge Philos. Soc. 65 (1969), 439-444.

[2] L. E. Dickson, History of the Theory of Numbers, Vol. II, first publ. in 1919. Reprint: Chelsea, New York, 1971.

[3] Ya. Gabovich, On arithmetic progressions with equal products of terms, Colloq. Math. 15 (1966), 45-48 (in Russian).

[4] Problèmes P543 et P545, R1, ibid. 19 (1968), 179-180.

[5] N. Saradha and T. N. Shorey, On the ratio of two blocks of consecutive integers, Proc. Indian Acad. Sci. Math. Sci. 100 (1990), 107-132.

[6] - - - On the equation $x\left(x+d_{1}\right) \ldots\left(x+(k-1) d_{1}\right)=y\left(y+d_{2}\right) \ldots\left(y+(m k-1) d_{2}\right)$, ibid. 104 (1994), 1-12.

[7] N. Saradha, T. N. Shorey and R. Tijdeman, On arithmetic progressions of equal lengths with equal products, Math. Proc. Cambridge Philos. Soc., to appear. 
[8] T. N. Shorey and R. Tijdeman, Perfect powers in products of terms in an arithmetical progression (II), Compositio Math. 82 (1992), 119-136.

SCHOOL OF MATHEMATICS

TATA INSTITUTE OF FUNDAMENTAL RESEARCH HOMI BHABHA ROAD

BOMBAY 400 005, INDIA
MATHEMATICAL INSTITUTE LEIDEN UNIVERSITY P.O. BOX 9512 2300 RA LEIDEN, THE NETHERLANDS 